\title{
AN EMPIRICAL APPROACH TO THE MENTAL STATUS EXAMINATION
}

\author{
BILLIE S. ABLES PhD
}

JEFFREY M. BRANDSMA, PhD GEORGE M. HENRY, MD

\begin{abstract}
The development of the Empirical Mental Status Exam is described. Its objectivity derives from observations of well-defined behavioral variables and from specific tests of cognitive functioning. Clear guidelines are offered for interpretation. Long usage indicates its utility as a clinical and teaching tool.
\end{abstract}

\section{INTRODUCTION}

In the practice of psychiatry, the Mental Status Exam (MSE) has long complemented the physical exam and history-taking as essential for assessing the integrity of a patient's emotional and mental functioning. Beginning in 1974 at the University of Kentucky Medical School, we set out to develop an instrument that would codify a high standard of practice and help organize our teaching program in various settings.

There are many different approaches to the MSE, but most agree about the general categories of behavior to be observed. ${ }^{1-13}$ The various MSE's however, vary greatly in form, content, and in their specificity of instructions and operations to be performed. Furthermore, they rarely provide normative standards for assessing degree of impairment. Those that cover a broad range of behavior tend to be very non-specific whereas those that are narrow focused (for

Dr. Ables is affiliated with the Department of Psychiatry, University of Kentucky Medical School. Dr. Brandsma is with the Department of Psychiatry, Medical College of Georgia. Dr. Henry is in private practice in Macon, Georgia. Requests for reprints and for materials mentioned in the text should be directed to Dr. Brandsma, Dept. of Psychiatry, Medical College of Georgia, Augusta, GA, 30912. 
Table 1. Outline of the Mental Status Exam
A. Appearance
B. "Broca's Area", i.e., Speech
C. Cognitive Functions
1. Orientation
2. Attention $\&$ Concentration
3. Memory
4. Abstraction
5. Perceptual-Motor and Language Functions
6. Intelligence
D. Discerment/Discretion
1. Judgement
2. Insight
E. Emotions
F. Fantasies/Thought/Perceptions

example; anxiety, depression scales) $)^{14-19}$ suffer on that account, that is, there is a power versus breadth problem.

Our Empirical Mental Status Examination (EMSE) attempted to remedy the disadvantages inherent both in rating scales and existing MSE's. As with the older MSE'S, it encompasses all major categories of behavior; as with rating scales, it provides a systematic method of collecting data. However, its overview is more comprehensive than most rating scales, it gives a consensual definition of terms, and it specifies procedures assessment, providing interpretive, normative guidelines when possible. 


\section{DEVELOPMENT}

In the early years of development, we organized the EMSE into nine categories of descriptive psychopathology and one category (that is; cognitive functions) of empirical procedures. Recently this approach to organization has been changed in order to make the procedures easier to teach and remember. The current protocol is organized on an alphabetical format with both descriptive and empirical procedures iterated (where appropriate) for each major concept to be evaluated. An outline of our protocol can be found in Table 1. In addition, a brief format based on the EMSE, but organized by an acronym, is provided in an appendix for those situations where a complete MSE is not possible.

The key aspect to what is unique about the EMSE is that specific procedures for operational definition are provided to assess orientation, atttention and concentration, memory (short and long), abstraction, perceptual-motor functioning, and judgment. We utilized several well known, standardized tests or parts of tests and created additional procedures patterned after certain sub-tests from the Wechsler Memory Scale ${ }^{20}$ and the Wechsler-Bellevue Scale. ${ }^{21}$ Where it was necessary to develop original tests, we collected our own standardization sample of 38 outpatients from the various medical clinics at the University of Kentucky Medical Center. The demographic and statistical characteristics of this sample and their scores on our newly devised tests are available from the authors. In sub-tests where comparisons of scores were possible, it was noted that this sample compared closely to Wechsler's. Interpretive statements for our new tests were based on the performance of our sample population, and the other interpretive statements were based on the previous research on those procedures. A more extensive sample of other populations of specific interest would be easy to obtain, since our procedures are easy to administer.

\section{DESCRIPTION OF COGNITIVE FUNCTION PROCEDURES}

The sub-test called "orientation" includes five items which measure orientation for time and place. Attention and concentration are assessed by two sub-tests called; (1) "Digit Retention" with "Digits Forward" (DF) and "Digits Backwards" (DB), and (2) "Serial Subtraction of Sevens" (SSS). These sub-tests represent a continuum of complexity in the order of DF, DB, and SSS. Items for DF and DB were generated from a table of random numbers. 
The SSS is utilized in the conventional form as it appears in a number of standard MSE's. A review of the literature indicates that this test has a high false-positive rate for any criteria of success when used with a normal population-even when the subjects have above average education, intelligence, and socioeconomic status. However, when this test is taken in context with other measures of attention and concentration (that is, DF and DB) and with the overall clinical picture, it adds an increment of complexity which may be diagnostically useful. For this reason, it was included in the EMSE. Interpretive suggestions were made on the basis of our sample and the previously published studies. ${ }^{22-24}$

Tests for the evaluaton of memory are threefold. "Information," and two forms of "Logical Memory"-"Immediate Recall," and "Delayed Recall". Information questions were selected which would be fairly easy and would relate to pertinent personal and current information. For immediate and delayed Logical Memory, the Babcock Story Recall is utilized with administration and scoring according to Rapaport et al. ${ }^{25}$

The items constructed to assess judgment were intended to be relatively easy. Thus, failure, on any of these items can be seen as reflecting impaired judgment rather than indicating limited education of experience.

For the procedure measuring ability to abstract, six items were utilized to assess facility with verbal concept formation. Two items each were chosen to represent easy, intermediate, and difficult tasks of abstraction. As an additional measure of abstract verbal functioning, six proverbs were included from the proverbs test (Form II) by Gorham. ${ }^{26}$ Since in Gorham's procedure the items are arranged in order of difficulty, we excluded alternate proverbs to shorten the test, but still retain the full range of difficulty. Interpretive norms are based on Gorham's system of scoring for abstraction.

The final sub-tests were aimed at providing a gross, screening measure for perceptual-motor and language dysfunction. These four tests, part of the larger Halstead-Wepman Aphasia Screening Test ${ }^{27}$ were shown effective in distinguishing right versus left hemispheric gross damage in 239 patients. ${ }^{28}$ Three of the four tests are designed to detect difficulty in copying geometric figures (right hemisphere). All four are used to detect dysfunction of language (left hemisphere). Guidelines for interpretation of data are based on the work of Heimburger and Reitan. ${ }^{28}$

In the early years of development, it was hoped that a quick measure of intelligence could be derived from some form of a 
vocabulary test, since these tests traditionally correlated highest with the overall IQ score. We tried a short form of the WAIS Vocabulary and the Quick Word Test, but these efforts proved to be too long in administration time to include them in the EMSE. Thus, in the last few years we began using the Rapid Adult Intelligence Test (RAIT) ${ }^{29}$, because of its ease of administration. It provides a gross measure of that most important clinical concept, intelligence, a notion of which should be included in all cognitive and behavioral assessment.

\section{DISCUSSION}

The EMSE has been found to be a useful instrument for making clinical assessment of a patient's behavior. The cognitive function subtests have the advantage of quantification, and norms or guidelines are provided for interpretation. We have found that a high degree of interrater reliability can be achieved through training on the descriptive sections using a four-page record booklet. In one attempt, second year medical students were given training and practical experiences in rating behaviors as presented to them on videotapes. Ratings by members of the faculty were available for immediate comparison, similar to the method described by Miller and his co-workers for the S.A.I.D. Program. ${ }^{30}$ When the students begin to work up new patients during the third year psychiatry clerkship, the residents and faculty who supervise them provide extensive further training in making the necessary judgements about specific aspects of behavior. The students' presentation of the EMSE is then reviewed by members of the faculty to compare with assessments which they have made. In addition, the clinical clerks have been given periodic exercises to compare their rating skills with each other and with the resident staff on more videotaped interviews.

For the first seven years of using the EMSE, over 600 third year medical students and 30 first year residents have administered it to almost all the psychiatric inpatients and outpatients whom they saw. With few exceptions, the instrument was felt to be helpful in assessing the patient. All the data required to write an accurate and complete record (with the help of a specialized form available from the authors) can be gathered within 30-45 minutes after completing a semistructured, standard psychiatric intake interview. A 17 page instruction booklet, together with a backlog of prior training, were sufficiently clear to allow ready administration of the tests from the beginning of the students' clerkship.

Impressions of faculty members who supervised the mental 
status evaluations by students and residents were similarly favorable. Without exception, the faculty thought initial presentations of new patients at case conferences and rounds were more orderly than before the instrument came into use. The student understood his patient better, because he paid attention to complex interrelationships between, for example, a disturbed patient's labile affect and his minimally impaired cognitive functions. The student became more aware that his own feelings in response to the patient provide important data about the patient. He had new categories for conceptualizing his patient and more data to "flesh out" these categories. In addition, students greatly appreciated the structure provided by the EMSE. Psychiatric examination was now seen as objective, scientific, and reproducible-a surprise to the often previously negatively biased view of many medical students that psychiatry was purely subjective. The information they obtained was now seen to be as identifiable as that obtained in the physical or neurological examinaiton. Another distinct advantage came from using the tests repeatedly when patients were in states of change. A patient who was thought to be clinically different from time to time was readministered the relevant subtest(s) by students or residents to identify and document the change(s).

The EMSE has been found to be very useful across the range of teaching opportunities in medical school and residency. The school year provides definition of various concepts in psychology and can be used as a review. Beyond that, complex concepts are operationally defined and the various deficits can be put into a schema of diagnostic categories rather easily. This relates operationally to the areas that medical students are used to thinking about and highly motivated to learn, that is, examination and diagnosis. In the clinical years, it provides a structure for remembering, organizing, and doing a competent assessment. It helps medical students appreciate the tests and procedures of psychologists and neuropsychologists. In the residency years, further refinements take place: well learned procedures can be taught effectively and used selectively, particularly in documenting deficits or changes; new procedures can be added to one's armamentarium. The value of empirical procedures is always appreciated in forensic applications.

In summary, the EMSE is rooted in the traditional MSE, but has added refinements and improvements. It is organized for teaching and remembering, yet systematic and quantifiable in its approach to the study of mental functioning and behavior. Because of its empirical orientation, it lends itself to further study and to use in teaching and research. It fits different functions in teaching for the second and third 
years of medical training, being refined in residency training. If used at all these levels, it provides for a consensual approach to using complex concepts competently and in building on one's experience. It is proposed as a valuable clinical instrument for teaching, psychiatric diagnosis, and assessing behavior.

\section{REFERENCES}

1. Redlich, F.C., Freedman, D.X.: The Theory and Practice of Psychiatry. New York, Basic Books, 1966.

2. Noyes, A.P., Kolb, L.C.: Modern Clinical Psychiatry. Philadelphia, W.B. Saunders Co., 1964.

3. Stevenson, I., Sheppe, W.: The psychiatric interview, in S. Ariete (ed): The American Handbook of Psychiatry. New York, Basic Books, 1959, pp. 215-234.

4. Storrow, H.A.: Outline of Clinical Psychiatry. New York, AppletonCentury-Crofts, 1969.

5. Sands, W.: Psychiatric History and mental status, in H.I. Freedman, A.M. Kaplan (eds.): Comprehensive Textbook of Psychiatry. Baltimore, Williams \& Wilkins, 1954, pp. 499-508.

6. Menninger, K.A.: A Manual for Psychiatric Case Study. New York, Grune and Stratton Inc., 1962.

7. Wells, F.L., Reusch, J.: Mental Examiners' Handbook. New York, The Psychological Corp., 1945.

8. Lewis, N.: Outlines for Psychiatric Examinations. Albany, The New York State Department of Mental Hygiene, 1943.

9. Engel, I., Goldstein, R., Thaler, O.: Mental Status Examination, Department of Psychiatry, University of Rochester Medical Center, 1964.

10. Lorr, M., Klett, C.H., McNair, D.M., Lasky, J.J: Inpatient MultiDimensional Psychiatric Scale Manual. Palo Alto California, Psychological Press, 1962.

11. Whittenborn, J.R.: Psychiatric Rating Scales. New York, Psychological Corp., 1955.

12. Overall, J.E., Gorham, D.R.: The brief psychiatric rating scale. 10:799$812,1962$.

13. Endicott, J., Spitzer, R.L.: What! Another rating scale? The psychiatric evaluation form. Journal of Nervous Mental Disorders. 54(2):88-104, 1972.

14. Grinker, R.R., Miller, J., Sabshin, M., Nann, R., Nunnally, J.C.: The Phenomena of Depressions. New York, Hoeber, 1961.

15. Hamilton, M.: A rating scale for depression. Journal of Neurology, Neurosurgery and Psychiatry 23:56-62, 1960.

16. Clyde, D.J.: Manual for the Clyde Mood Scale. Biometric Laboratory, University of Miami, Coral Gables, Florida, 1963.

17. Beck, A.T., Ward, C.H., Mendelson, M., Mock, J., Ebaugh, J.: An inventory for measuring depression. Archives of General Psychiatry. 4:561$571,1961$. 
18. Bunny, W.E., Hamburg, D.: Methods for reliable longitudinal observation of behavior. Archives of General Psychiatry, 9:280-294, 1963.

19. Beigel, A., Murphy, D.L., Bunny W.E., Jr.: The manic state rating scale. Archives of General Psychiatry 25:256-262, 1971.

20. Wechsler, D.: A standardized memory scale for clinical use. Journal of Psychiatry 19:87-95, 1945.

21. Wechsler, D.: Measurement of Adult Intelligence. Baltimore, Waverly Press Inc., 1955.

22. Milstein, V., Small, J., Small, I.: The subtraction of serial sevens test in psychiatric patients. Archives of General Pschiatry 26:439-441, 1972.

23. Smith, A.: The serial sevens subtraction test. Archives of Neurology 17:78-80, 1967.

24. Hayman, M.: The use of serial sevens in psychiatric examination. American Journal of Orthopsychiatry 11:334-355, 1941.

25. Rapaport, R., Gill, M., Schafer, R.: Diganostic Psychological Testing. New York, Internatioal Universities Press, 1968.

26. Gorham, D.R.: A proverbs test for clinical and experimental use. Psychology Rep. Monogram Supplement, 1956, No. 1.

27. Halstead, W.C., Wepman, J.M.: The Halstead-Wepman aphasia screening test. Journal of Speech Disorders 14:9-15, 1949.

28. Heimburger, R.F., Reitan, R.H.: Easily administered written test for lateralizing brain lesions. Journal of Neurosurgery 18:301-312, 1961.

29. Wilson, I.C.: Rapid Approximate Intelligence Test. American Journal of Psychiatry 123: 10:1289-1290, 1967. 